\title{
VCSEL-based communications for metro and access networks
}

\author{
Alberto Gatto \\ Dipartimento di Elettronica, Informazione e Bioingegneria \\ Politecnico di Milano, Milano, Italy \\ alberto.gatto@polimi.it \\ Paolo Martelli \\ Dipartimento di Elettronica, Informazione e Bioingegneria \\ Politecnico di Milano, Milano, Italy \\ paolo.martelli@polimi.it
}

\author{
Paola Parolari \\ Dipartimento di Elettronica, Informazione e Bioingegneria \\ Politecnico di Milano, Milano, Italy \\ paola.parolari@polimi.it \\ Pierpaolo Boffi \\ Dipartimento di Elettronica, Informazione e Bioingegneria \\ Politecnico di Milano, Milano, Italy \\ pierpaolo.boffi@polimi.it
}

\begin{abstract}
The capabilities of long-wavelength directlymodulated VCSELs for applications in sustainable metro and access networks are reported. 25-Gb/s transmission is demonstrated thanks to asymmetrical filtering and KramersKronig receiver for passive optical networks, while VCSEL exploitation together with coherent detection in metro network is envisaged.
\end{abstract}

Keywords-Vertical cavity surface emitting laser, direct modulation, passive optical networks, metro networks

\section{INTRODUCTION}

In recent years, the continuous growth of bandwidth demand in the access and metro optical networks is pushing towards a line rate increase beyond $10 \mathrm{~Gb} / \mathrm{s}$. In the access scenario, the challenge is to perform the next upgrade of the optoelectronic front-ends in a cost-effective way by using limited-bandwidth direct-detection (DD) receivers [1], while maintaining the compliancy with previous standards, as they are conceived as long-term evolutions for network operators. Specifically, the optical network unit transmitters have to be colorless, preferably tunable over 4-8 times the grid-spacing, with very high side-mode suppression ratio (SMSR) to avoid crosstalk into neighboring channels. Moreover, further benefits could be gained by extending the optical reach to about $100 \mathrm{~km}$ : the reduction of the number of network nodes, eliminating electronic equipment for traffic aggregation, routing, and switching will in fact reduce both cost and energy consumption [2]. The proposal of a multi-wavelength longreach (LR) PON with data rates higher than $10-\mathrm{Gb} / \mathrm{s}$ will thus increase the long-term economic viability and sustainability of a next-generation network deployment. On the other hand, the current transmission devices for metro network are evolving from the traditional approach by proposing enhanced modulation schemes or, as alternative, coherent transmission solution, which today are used mainly for long haul transmission and are characterized by high cost and highpower consumption. With these approaches, the expected performance enhancement in terms of throughput is limited to a factor $\mathrm{x} 2-\mathrm{x} 4$, which seems to be inadequate to support $5 \mathrm{G}$ and beyond network requirements. Moreover, a migration towards more flexible, efficient and agile paradigms is expected, owing to the aggregation of the metro/access networks and for the support of heterogeneous networks. In this context, the programmable and modular sliceable bandwidth/bitrate variable transceiver (S-BVT) represents an attractive solution, when suitably tailored for this network segment [3]. Particularly, the adaptability/reconfigurability of multiple parameters by software defined networking (SDN), combined with a modular approach, allows flexible adaptation to the path/traffic condition with wide range granularity, while saving network resources and costs.

Photonic technologies can provide new energy- and costefficient solutions, starting from the adoption of direct modulation (DM) of laser sources; among them, vertical cavity surface emitting lasers (VCSELs) could allow a radical reduction of transmitter cost, power consumption and footprint. Up to now, VCSEL exploitation has been considered mainly for datacom applications in the first window of the optical communications. Recently, their potential has been shown even at $1550 \mathrm{~nm}$ [4] for $100 \mathrm{G}$ applications as well as for passive optical networks (PONs) and programmable and modular sliceable bandwidth/bitrate variable transceivers-based metro networks [5].

To achieve high transmission bit rates, advanced modulation formats have been proposed in order to exploit limited bandwidth devices. In particular, multicarrier modulation, as discrete multitone (DMT), can enable spectral manipulation leading to an efficient usage of the bandwidth resource. Thanks to bit and power loading techniques, dynamic and flexible adaptation to traffic/channel conditions can be reached reducing the spectrum fragmentation issue in metro networks [3].

In this paper, we show the capabilities of long-wavelength VCSELs for applications in sustainable metro and access networks demonstrating transmission rates higher than 25 $\mathrm{Gb} / \mathrm{s}$ for fiber lengths compliant with metro-access distances. In particular, we demonstrate the capabilities of a tunable transmitter, presenting low-cost and reduced footprint, together with DMT modulation. Moreover, the exploitation of Kramers-Kronig (KK) detection [6] for full electronic dispersion compensation is considered to target high capacity for an extended-reach access network. Finally, directlymodulated long-wavelength VCSELs together with S-BVT and coherent detection exploitation in metro networks is envisaged.

\section{VCSEL-BASED SOLUTIONS FOR DiRECT-DETECTED EXTENDED-REACH ACCESS NETWORKS}

\section{A. Long Wavelength VCSELs}

Recently, long-wavelength directly-modulated VCSELs showed their capabilities for low-cost and low-complexity short-reach connections. Their exploitation has been demonstrated both for standard modulation formats (as onoff keying or pulse amplitude modulation) [7] and advanced modulation formats (like DMT or frequency division multiplexing transmission) $[4,8]$ to reach very high capacity 


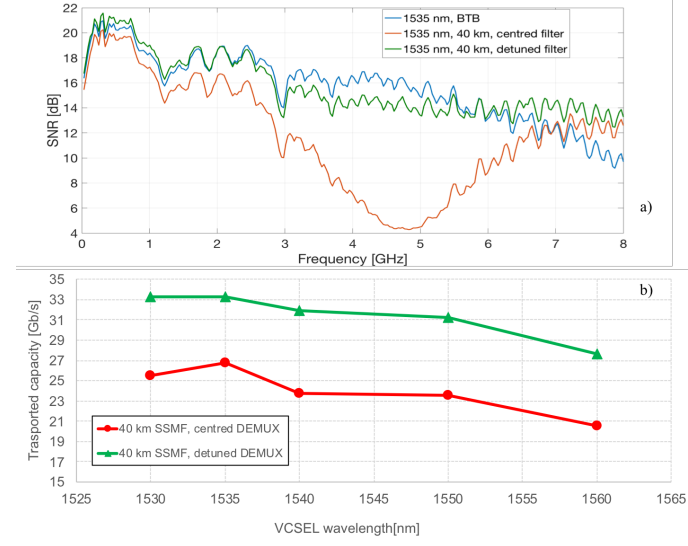

Fig. 1. a) Measured SNRs at $1535 \mathrm{~nm}$ for back-to-back condition (blue curve), after 40-km SSMF propagation with centered filter (red curve) and detuned filter (green curve). b) Total capacity transported by the system with centered (blue line, circles) and detuned (orange line, squares) filter The reference capacity of $25 \mathrm{~Gb} / \mathrm{s}$ is the green dotted line.

transmission over few kms of standard single-mode fiber (SMF). The Buried Tunnel Junction (BTJ) VCSELs feature a very high bandwidth over temperature, supporting data rates up to $50 \mathrm{~Gb} / \mathrm{s}$, a low threshold current of around $1-2 \mathrm{~mA}$ and an operating voltage of 1.5 to $1.7 \mathrm{~V}$. Moreover, single-mode widely tunable long-wavelength BTJ VCSELs featuring a MEMS top mirror can be thermo-electrically controlled, obtaining a mode-hop free tuning range of about $90 \mathrm{~nm}$ (1517 $\mathrm{nm}$ to $1608 \mathrm{~nm}$ ), with more than 45-dB SMSR and S-21 3$\mathrm{dB}$ bandwidth up to $7 \mathrm{GHz}$ at $1550 \mathrm{~nm}$ [9].

\section{B. Beyond $25 \mathrm{~Gb} / \mathrm{s}$ Next-Generation Access Networks}

Thanks to multicarrier modulation, like DMT, the spectral efficiency of the optical channel can be drastically increased even using directly-modulated sources as VCSELs. However, frequency chirp can strongly limit system performance, owing to the frequency fading generated by the interaction between VCSEL frequency chirp and the cumulated chromatic dispersion (CD). Even if its impact can be mitigated by adaptive subcarrier loading, a drastic reduction of the transported capacity is expected. A promising solution for future PONs and limited reach (e. g. few tens of $\mathrm{km}$ ) could be the exploitation of DMT transmission together with asymmetrical filtering, in order to achieve capacities beyond $25 \mathrm{~Gb} / \mathrm{s}$ even with optical devices suitable for $10-\mathrm{Gb} / \mathrm{s}$ transmissions [8]. As a proof of principle, we demonstrate the feasibility of a directlymodulated tunable VCSEL characterized by a limited electrooptical bandwidth of $7 \mathrm{GHz}$ without any $\mathrm{CD}$ compensation, checking its performance over the entire C-band with and without asymmetrical filtering.

At first, the estimation of the channel characteristics has been performed by transmitting a probe DMT signal mapped with uniform QPSK loading, providing the signal-to-noise ratio (SNR) of each sub-carrier after DD. An example of measured SNRs in case of back-to-back (BTB) condition, 40$\mathrm{km}$ SSMF propagation with centered filter and 40-km SSMF propagation with detuned filter is shown in Fig. 1a) at 1535 $\mathrm{nm}$. After $40 \mathrm{~km}$ of SSMF propagation (red curve), the cumulated chromatic dispersion leads to an evident frequency dip around $5 \mathrm{GHz}$, where the corresponding SNR shows a minimum of about $4 \mathrm{~dB}$. Thanks to the filter detuning (green curve), the impact of chromatic dispersion is mitigated, leading to an almost unchanged SNR response with respect to the BTB condition and, hence, to a strong enhancement of the achievable capacities on the entire C-band, as shown in Fig. 1b). In case of filter detuning, in fact, capacities higher than $27 \mathrm{~Gb} / \mathrm{s}$ are achieved, while on the other hand, when the filter is centered to the VCSEL emission, the transported capacity varies between $27 \mathrm{~Gb} / \mathrm{s}$ at $1535 \mathrm{~nm}$ and $20 \mathrm{~Gb} / \mathrm{s}$ at $1560 \mathrm{~nm}$.

\section{Extended-Reach Access Networks based on Kramers- Kronig direct detectors}

To cope with the chromatic dispersion issue in extendedreach PONs, single sideband (SSB) transmission could be considered, thanks to its greater resilience to $\mathrm{CD}$; however, the exploitation of direct modulation, adding a substantial chirp, and the necessity to perform vestigial sideband (VSB), i.e. optically performing SSB, could limit SSB effectiveness [10]. Another promising solution is the exploitation of $\mathrm{KK}$ detection, which allows the optical field reconstruction by a suitable digital signal processing (DSP) after DD and, hence, the CD compensation. Moreover, the spectrum of DM lasers with multicarrier signals naturally presents a strong carrier component. For exploiting KK processing, hence, only an optical filter to achieve the SSB condition is mandatory [6].

To demonstrate the capabilities of directly-modulated tunable VCSELs together with DMT and KK detectors for extended-reach PONs we performed suitable experiments. In particular, the VCSEL is directly modulated by a DMT signal composed by 256 sub-carriers in $10 \mathrm{GHz}$ range. The performance of the system has been measured in case of standard dual side-band (DSB) transmission and for a KKbased receiver after direct detection. In case of DSB transmission, a modulation amplitude of $950 \mathrm{mV}$ is applied, while we choose the trade-off of limiting the modulation amplitude to $450 \mathrm{mV}$ in case of KK-based transmissions for fulfilling the minimum-phase condition [6].
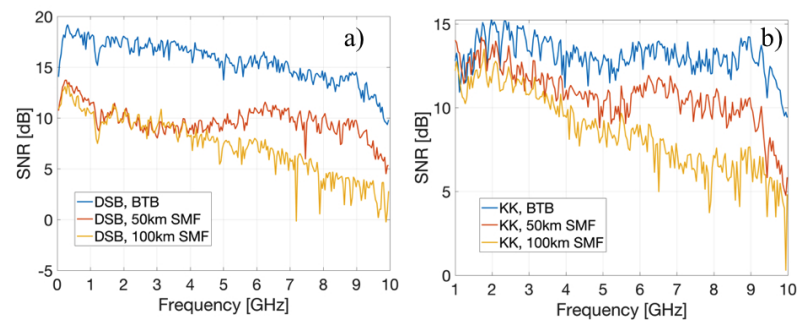

Fig. 2. Measured SNRs at $1545 \mathrm{~nm}$ for BTB condition (blue curve), after 50$\mathrm{km}$ (red curve) and 100-km SMF propagation (orange curve) for a) DBS and b) KK-based transmission systems.

The optical signal is transmitted over up to $100-\mathrm{km}$ uncompensated SMF. At the receiver end, direct detection by a standard PIN receiver suitable for $10-\mathrm{Gb} / \mathrm{s}$ operation has been exploited to maintain the compliancy with PON standards.

An example of signal-to-noise ratios (SNR) provided for each sub-carrier in case of back-to-back (BTB) condition, 50$\mathrm{km}$ SMF propagation and $100-\mathrm{km}$ SMF propagation is shown in Fig. 2 at $1545 \mathrm{~nm}$ for both the configurations under test. In particular, a SNR degradation is noticeable for DSB transmission, for $50 \mathrm{~km}$ at lower frequencies and then on the whole signal spectrum due to the interplay between VCSEL frequency chirp and cumulated CD (Fig 2a)). For KK-based transmission, a SNR equalization is obtained in BTB and after 50-km SMF propagation, thanks to the optical filtering; however, due to the filtering of one sub-band, the SNR 
absolute value is reduced of at least $2 \mathrm{~dB}$ (Fig. 2b)). After 100$\mathrm{km}$ propagation, the SNR is reduced at higher frequencies. The measured SNRs are exploited for performing Chow's algorithm, setting the target bit error rate (BER) at $4.62 \cdot 10^{-3}$. The experimentally obtained capacities are reported in Tab. I.

TABLE I. MEASURED TRANSPORTED CAPACITIES IN CASE OF DSB AND KK-BASED DMT TRANSMISSION

\begin{tabular}{|c|c|c|}
\hline & \multicolumn{2}{|c|}{ Capacity [Gb/s] } \\
\hline SMF [km] & DSB & KK \\
\hline 0 & 37.3047 & 26.4453 \\
\hline 50 & 16.25 & 21.4453 \\
\hline 100 & 9.0875 & 15.625 \\
\hline
\end{tabular}

\section{LONG-WAVELENGTH VCSELS FOR METRO NETWORKS}

To face the expected traffic demand growth in the metro network a further leap forward is demanded as target distances and capacities are beyond 100 kilometers and $25 \mathrm{~Gb} / \mathrm{s}$ respectively. Directly modulated VCSELs should be combined with coherent detection to provide an effective solution, featuring an efficient usage of the optical spectrum, maximizing capacity, fulfilling scalability, flexibility and endto-end performance. This approach is carried forward by the European project PASSION, which is proposing a metro network architecture based on innovative photonic technologies, novel transmission techniques, flexible switching solutions and dynamically configured softwaredefined functionalities.

In the envisaged metro network, arrays of VCSEL-based modules are projected to generate a sliceable bandwidth/bitrate variable transmitter (S-BVTx). The transmitter can be suitably programmed to adaptively generate multiple flows targeting $\mathrm{Tb} / \mathrm{s}$ capacities to meet the traffic demand, channel bandwidth/path/state and energy efficiency requirements of the metro network. In particular, the programmable and modular S-BVT consists of an array of $N$ BVTs with 25-GHz spacing. The BVT array can be composed of subsets of BVT modules (enabling a scalable pay-as-yougrow model based on pluggable units and photonic integration), whose BVTx front-end are based on DM VCSELs covering the entire C-Band. The receiver will be based on a simplified coherent detector for achieving electronic dispersion compensation and polarization multiplexing, while phase and frequency carrier recovery can be avoided since the retrieval of the lone signal intensity is mandatory. The multiple slices can be transmitted over the network as a single high-capacity flow or can be split into lower capacity flows routed towards independent paths [3].

The array of modules of the S-BVTx will be based on the high-speed, low-energy consumption, high-reliability and low-cost InP-based VCSELs described in the previous paragraphs, emitting in the C-band and characterized by an electro-optical bandwidth of more than $20 \mathrm{GHz}$. Each VCSEL will be directly modulated with multicarrier formats (e.g. DMT) in order to adaptively load/manipulate the spectrum at subwavelength granularity level to achieve up to $50 \mathrm{~Gb} / \mathrm{s}$. Four BVT modules can be aggregated in order to support up to $8 \mathrm{~Tb} / \mathrm{s}$ capacity per state of polarization (Fig. 3). The aggregation and multiplexing of the VCSELs will be enabled by the development of compact $\mathrm{SiPh}$ integration solutions, giving significant target outcomes in terms of footprint and energy-efficiency.

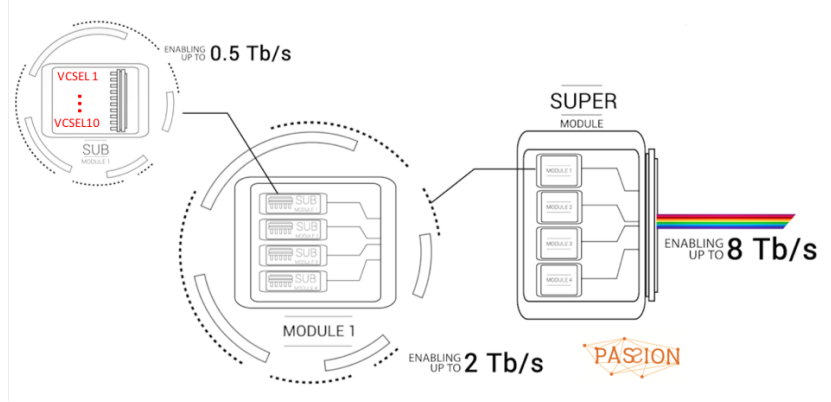

Fig. 3. Example of Tx modules enabling variable aggregated capacities, exploiting multiple dimensions.

\section{CONCLUSIONS}

The capabilities of directly-modulated long-wavelength VCSELs for short- and medium-reach transmission has been reported. The exploitation of widely-tunable VCSELs for next-generation access networks has been demonstrated to realize $25-\mathrm{Gb} / \mathrm{s}$ transmission thanks to multicarrier modulation formats like DMT and asymmetrical filtering to limit the impact of native frequency chirp. The exploitation of Kramers-Kronig detection to electronically compensate cumulated chromatic dispersion could also allow their employment in extended-reach access networks. The development of 20-GHz VCSEL to be exploited in SiPh modules, joined to coherent detection is envisaged by the European project PASSION for achieving up to $8 \mathrm{~Tb} / \mathrm{s}$ capacity per polarization. The exploitation of wavelength, bandwidth and space dimensions can further target an aggregated capacity per optical link beyond $100 \mathrm{~Tb} / \mathrm{s}$.

\section{ACKNOWLEDGMENT}

This work has been supported by the H2020 EU PASSION Project (GA 780326).

\section{REFERENCES}

[1] D. T. van Veen, V. E. Houtsma "Proposals for Cost-Effectively Upgrading Passive Optical Networks to a 25G Line Rate," J. Lightwav. Technol., 35, 1180 (2017).

[2] M. Ruffini, et al., "Deployment Strategies for Protected Long-Reach PON," IEEE/OSA Journal of Optical Communications and Networking, 4, 118-129, 2012.

[3] M. Svaluto Moreolo et al. "SDN-enabled Sliceable BVT Based on Multicarrier Technology for Multi-Flow Rate/Distance and Grid Adaptation,” J. Lightwav. Technol., 34, 8, 1516-1522 (2016).

[4] A. Gatto, D. Argenio, P. Boffi, "Very high-capacity short-reach VCSEL systems exploiting multicarrier intensity modulation and direct detection," Opt. Expr., 24, 12, 12769-12775 (2016).

[5] M. Svaluto Moreolo, et al., "Modular SDN-enabled S-BVT Adopting Widely Tunable MEMS VCSEL for Flexible/Elastic Optical Metro Networks," in OFC Conference, 2018, paper M1A.7.

[6] A. Mecozzi, C. Antonelli, and M. Shtaif, "Kramers-Kronig coherent receiver," Optica, 3, 1220-1226 (2016).

[7] S. Spiga et al.., "Single-Mode High-Speed 1.5- $\mu \mathrm{m}$ VCSELs," J. Lightwav. Technol., vol. 35, no. 4, pp. 727-733, Feb.15, 152017.

[8] A. Gatto, P. Parolari, C. Neumeyr, and P. Boffi, "Beyond $25 \mathrm{~Gb} / \mathrm{s}$ Directly-Modulated Widely Tunable VCSEL for Next Generation Access Network," in OFC Conference, 2018, paper Th1E.2.

[9] S. Paul et al., "10-Gb/s Direct Modulation of Widely Tunable 1550-nm MEMS VCSEL”, IEEE J. Sel. Top. Quant. Electr., Vol. 21, No. 6, p. 1700908 (2015)

[10] D. Pilori, C. Fludger and R. Gaudino, "Comparing DMT Variants in Medium-Reach 100G Optically Amplified Systems," J. Lightwave Technol., 34, 3389-3399, 2016. 